\title{
Free-shape 3D Structure Formation in Bulk Fused Silica by Irradiation with Femtosecond LaserPulses
}

\author{
Valdemar Stankevič ${ }^{* 1,2}$, Gediminas Račiukaitis ${ }^{1}$ \\ ${ }^{* 1}$ Center for Physical Sciences and Technology, Savanoriu Ave. 231, LT-02300, Vilnius, Lithuania \\ ${ }^{* 2}$ ELAS UAB, Savanoriu Ave. 231, LT-02300, Vilnius, Lithuania \\ valdemar.s@e-lasers.com
}

In this manuscript, the development of the method for formation of three-dimensional (3D) structures etched in bulk fused silica is presented. 3D structures according to the designed 3D CAD drawing were inscribed inside the transparent material and etched with hydrofluoric acid. At first, two different scanning strategies were used to investigate the volume etching rate. The main technique for the formation of three-dimensional structures was based on the laser direct writing by scanning the multiple line arrays slice by slice inside fused silica to obtain rectangular channels. Complex 3D structures were formed by applying different scanning strategies in combination with linear and circular polarization and different pulse energies when the laser fluence was sufficient to induce the type-II modification in fused silica.

DOI: $10.2961 / \mathrm{jlmn} .2014 .03 .0018$

Keywords: Femtosecond pulses, direct laser writing, fused silica, selective chemical etching, scanning strategy, internal modification

\section{Introduction}

The nowadays trends to miniaturization of micro-fluidic and photonic devices demand application of new and promising techniques for the formation of micro components. The femtosecond laser microfabrication of transparent materials opens a new opportunities in fabrication of microfluidic, opto-fluidic or complex microoptomechanical devices $[1,2]$. The microfluidic devices can integrate different and complex functions for low reagent consumption, fast analysis and ease of operation and are of great interest in analytical chemistry and biomedical applications [3, 4]. By combining the fluids and light, a huge amount of creative optofluidic devices such as a micro-fluidic laser [5] or an optofluidic microchip composed of plano-convex lenses, optical waveguide and a micro-fluidic microchamber integrated in a single substrate [6] can be fabricated. A more detailed review of optofluidic and photonic devices is described in $[7,8]$.

The direct laser writing technique [9] using femtosecond laser pulses offers flexible opportunities compared with conventional fabrication methods of optofluidic and microfluidic devices such as soft lithography, injection molding or photolithography that require stacking and bonding layers to construct 3D structures. Femtosecond lasers can space-selectively induce local modifications in any transparent materials due the strong nonlinear absorption [10]. These internal modifications in fused silica cause the structural and chemical changes $[11,12]$. The modified regions can be selectively removed by immersing the samples to aqueous solutions of etchant such as hydrofluoric acid $(\mathrm{HF})$ or potassium hydroxide $(\mathrm{KOH})$ [13] resulting in direct fabrication of the true 3D micro-devices inside fused silica[11]. Therefore, the whole microfabrication process consists of two steps [12]: 1) femtosecond laser direct writing and 2) chemical etching of modified regions.

In this manuscript, development of the technology for the formation of three-dimensional structures in bulk fused silica is presented. Two different 3D free-shape CAD drawings were prepared for different microfluidic applications. The embedded micro rotor rotating about its axis inside the etched cavity was demonstrated.

The main method used for structures formation is based on microchannels formation inside fused silica, where the micro-channels are formed with the laser direct writing technique by scanning the multi-line arrays for a rectangular channel [14] and the laser fluence was sufficient to induce the type-II modification in fused silica $[15,16]$. Two different scanning strategies in combination with control of the polarization orientation, laser pulse energies and scanning velocity were implemented in order to investigate the etching rate and surface morphology of complex $3 \mathrm{D}$ structures.

\section{Experimental}

The results presented in this report were obtained with an experimental setup shown in Fig. 1. Rectangular samples $\left(15 \times 10 \times 2 \mathrm{~mm}^{3}\right)$ of optically-polished ultraviolet grade fused silica (JGS1) were irradiated with ultrashort laser pulses with the pulse duration of $290 \mathrm{fs}$ at the repetition rate of $100-500 \mathrm{kHz}$ delivered from the diode-pumped 
mode-locked $\mathrm{Yb}: \mathrm{KGW}$ laser Pharos (Light Conversion, Ltd.) working at second harmonics $(\lambda=515 \mathrm{~nm})$. The pulse energy was varied with a motorized attenuator (half wavelength phase plate with two Brewster angle polarizers).

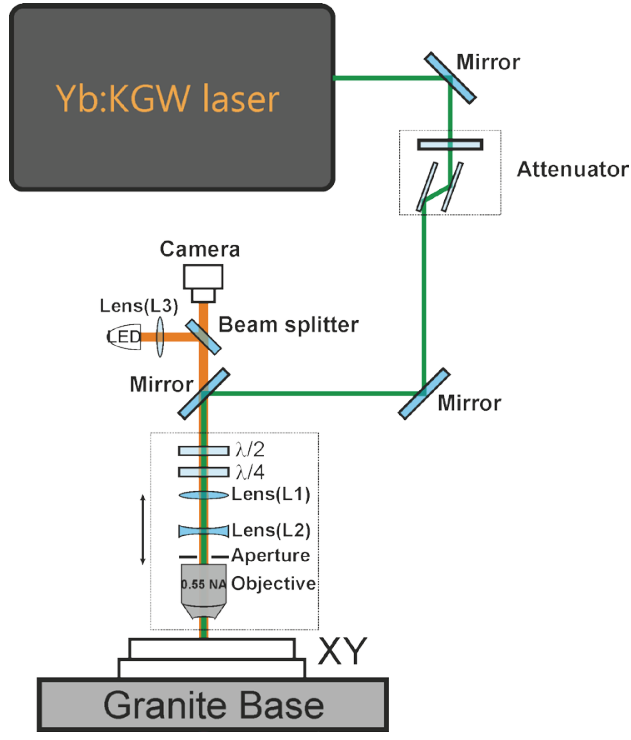

Fig. 1 Experimental setup for 3D structure formation.

The 50x Olympus objective with a 0.55 numerical aperture (NA) was used to focus laser beam and induce modifications inside fused silica samples (spot diameter at the focal position was $<2 \mu \mathrm{m}, 1 / \mathrm{e}^{2}$ level). For the sample positioning, the Aerotech XY linear axes (Aerotech ANT 130$160 \mathrm{XY}$ )with the $\sim 1 \mu \mathrm{m}$ accuracy were used. The focal position in the vertical direction was adjusted with the $\mathrm{Z}$ axis mounted on a granite bridge. The vision system with a CCD camera, lighting diode and a microscope objective was used to test the fabrication positioning on a sample. For polarization control, the zero-order $\lambda / 2$ and $\lambda / 4$ phase plates were used.

\section{Results}

In order to get a true 3D structure in transparent materi$\mathrm{al}$, the prepared 3D drawing was sliced into layers in $\mathrm{z}$ direction. The distance between slices was kept constant during the experiments $(3.5 \mu \mathrm{m})$. Two different scanning strategies - lines and contour scanning - were used to investigate the etching rate (Fig. $2 \mathrm{a}, \mathrm{b}$ ). The distance $\boldsymbol{d} \boldsymbol{l}$ between scanning lines was changed from 2 to $3 \mu \mathrm{m}$ during experiments.

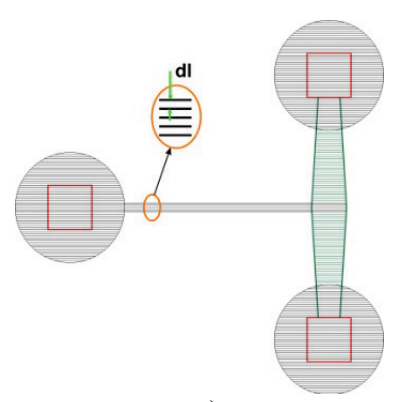

a)

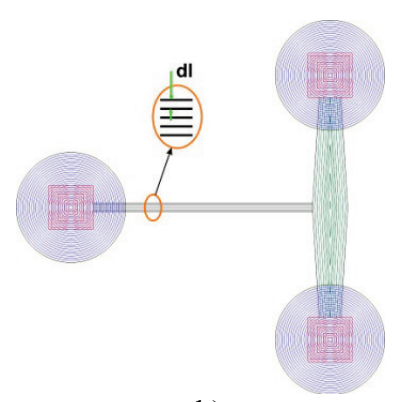

b)
Fig. 2 The principle of 3D micro pump formation: a) Lines scanning strategy ( $\boldsymbol{d} \boldsymbol{l}$ - distance between scanned lines); b) contour scanning strategy ( $\boldsymbol{d} \boldsymbol{l}$ - distance between contours).
The microstructures (Fig. 3) were formed using different laser pulse energies. The laser pulse energy was changed in the processing window where the nanogratings can be formed as was shown in [14] to achieve the highest etching rate. The micropumps were formed using pulse energy $200 \mathrm{~nJ}, 400 \mathrm{~nJ}$ and $600 \mathrm{~nJ}$ and linear polarization perpendicular to the scan ( $\boldsymbol{x}$ axis) direction and using pulse energy of $400 \mathrm{~nJ}$ and $600 \mathrm{~nJ}$ with circular polarization.

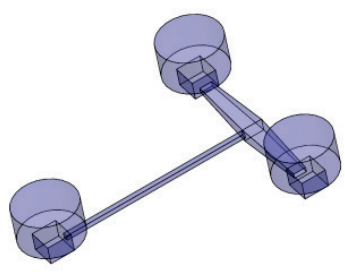

a)

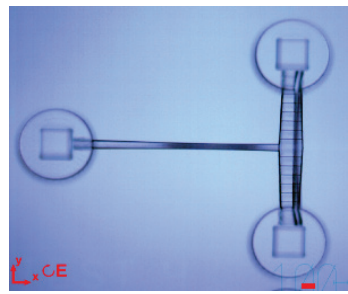

b)
Fig. 3a) 3D model of micropump; b) microstructure modified with the femtosecond laser in fused silica and etched in $10 \% \mathrm{HF}$ acid for $13.5 \mathrm{~h}$. Red bar indicates $100 \mu \mathrm{m}$.

The scanning was performed by the contour scanning strategy that facilitates the penetration of HF acid to the modified region (the polarization was maintained perpendicular to the $\mathrm{x}$ axis direction). In case of contour scanning, the acid always penetrates to the modified region along the scan line direction, and for line scanning, the acid can penetrate perpendicular or along the scanning line depending on the structure orientation. The etched volume for micropumps fabricated with different writing parameters was inspected by immersing the sample in $10 \% \mathrm{HF}$ acid for 13.5 hours. After 6 and 10 hours, the sample was pulled out and the removed volume was registered. Fig. 4 shows the dependence of the normalized etched volume on the etching time. Some micropump structures were fully etched only after 13.5 hours of etching and fluid transportation through the hollow volume was implemented.

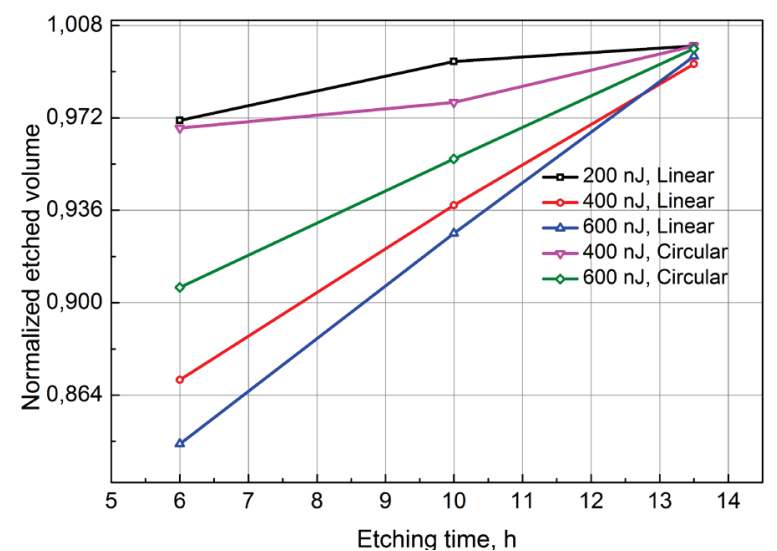

Fig. 4 Dependence of the etched volume of the micropump structures formed with the contour scanning on the etching time when linear and circular polarizations were used. The total volume of a micropump was $\sim 0.79 \mathrm{~mm}^{3}$.

The maximum etching rate was achieved for the structures fabricated using pulse energy of $200 \mathrm{~nJ}$ (for linear polarization) and $400 \mathrm{~nJ}$ (for circular polarization). As can be seen from Fig. 4, the etched volume in the samples fabricated with the circular polarization and $400 \mathrm{~nJ}$ pulse energy after $6 \mathrm{~h}$ was $\sim 11 \%$ larger and with $600 \mathrm{~nJ}$ pulse en- 
ergy $\sim 9.3 \%$ larger compared to fabrication with the linear polarization at the same conditions. This dependence shows that circular polarization can improve the etching time for the complex 3D microstructures. The presented results are in good agreement with the data reported in [17]. However, the circular polarization in combination with the contour scanning method is a new way how to improve the acid penetration into the complex structures. The processing with the pulse energies $<600 \mathrm{~nJ}$ generates the nanogratings inside the bulk material without microvoid formation [15] that enhances the acid penetration into the modified material and, in this way, the higher etching rate was achieved. As was discussed before, the line scanning of the complex structures obstructs acid penetration into the modified region as nanogratings orientation is optimized only for a single scan direction. To solve this problem, the contour scanning strategy was proposed. Fig. 5 shows the difference between micropump structures fabricated and etched with linear and contour scanning strategies.

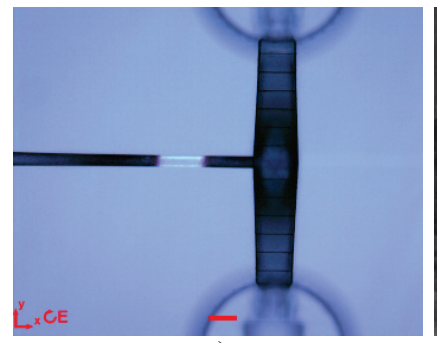

a)

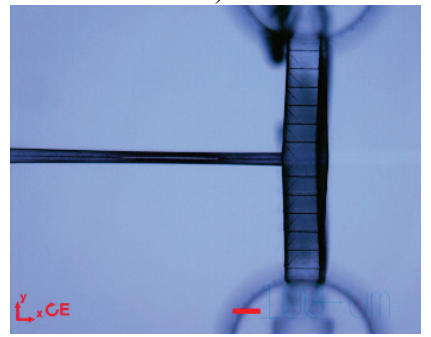

c)

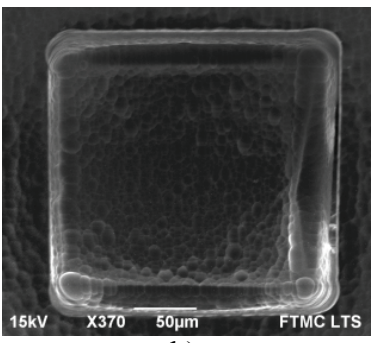

b)

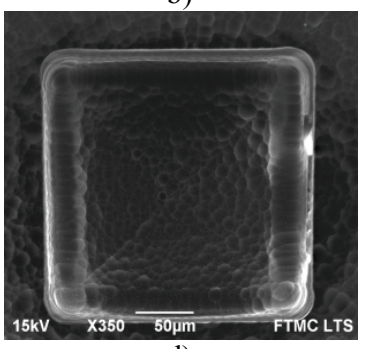

d)
Fig. 5 The micro pumps formed with: the line scanning strategy, a) optical microscope picture, b) SEM micrograph of enlarged etched area; and the contour scanning strategy, c) optical microscope picture, d) SEM micrograph of enlarged etched area. $(\mathrm{dl}=2$ $\mu \mathrm{m}, \mathrm{v}=1 \mathrm{~mm} / \mathrm{s}, \mathrm{E}=400 \mathrm{~nJ}$, circular polarization). Red bar indicates $100 \mu \mathrm{m}$.

The surface of the etched devices showed the semispherical shape of grain-like morphology. Depending on the processing energy, the grains were of a different size. The orientation of the grains depended on the scanning directions as can be seen from SEM pictures in Fig. $5 \mathrm{~b}$ and d. During the experiment, the grain dimensions were measured after $13.5 \mathrm{~h}$ of etching and averaged in three different samples processed with $200 \mathrm{~nJ}, 400 \mathrm{~nJ}$ and $600 \mathrm{~nJ}$ with linear polarization and in two samples processed with $400 \mathrm{~nJ}$ and $600 \mathrm{~nJ}$ for the circular polarization. Fig. 6 shows the grains diameters (sizes) dependence on the fabrication pulse energy.

The devices fabricated with the lower pulse energy have grains of a smaller size. The grains that correspond to the fabrication with circular polarization are larger than that get with linear polarization. It follows from the results that the fabrication with a lower pulse energy is necessary to minimize roughness of the etched surface without any post processing of the etched devices. The pulse energy that corresponds to the type II modification threshold should be applied. Anyway, annealing of the samples is required to achieve a smooth surface for micro optical devices fabrication [18].

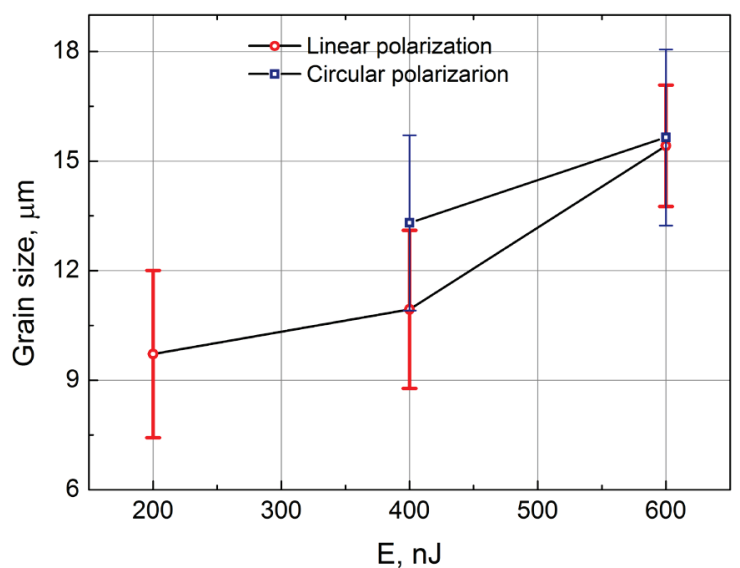

a)

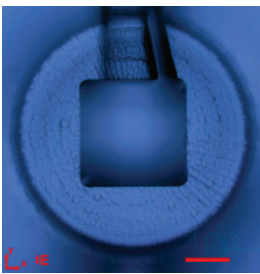

b)

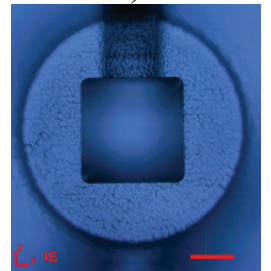

c)

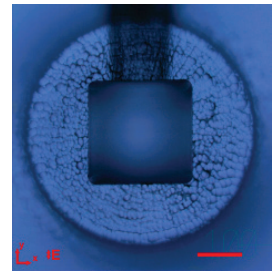

d)
Fig. 6 The grain size achieved after $13.5 \mathrm{~h}$ etching depending on the fabrication energy (a); microstructures fabricated with linear polarization and contour scanning strategy: b) $200 \mathrm{~nJ}$; c) $400 \mathrm{~nJ}$; d) $600 \mathrm{~nJ}$. Red bar indicates $100 \mu \mathrm{m}$.

To find out the morphology of the formed micropump, cross-section the sample was cleaved in the yz plane and the SEM analysis was performed (Fig. 7). As can be seen from the figure, the sharp edges of the microstructure can be achieved. From the enlarged microchannel, it is evident, that almost perfect rectangular $\sim 40 \times 55 \mu^{2}$ cross-section was achieved that can be used for the very low volume fluid pumping and transportation. The rough surface of the bottom of a microchannel demonstrates the scanning line orientation and shows that not all the modified material was removed.

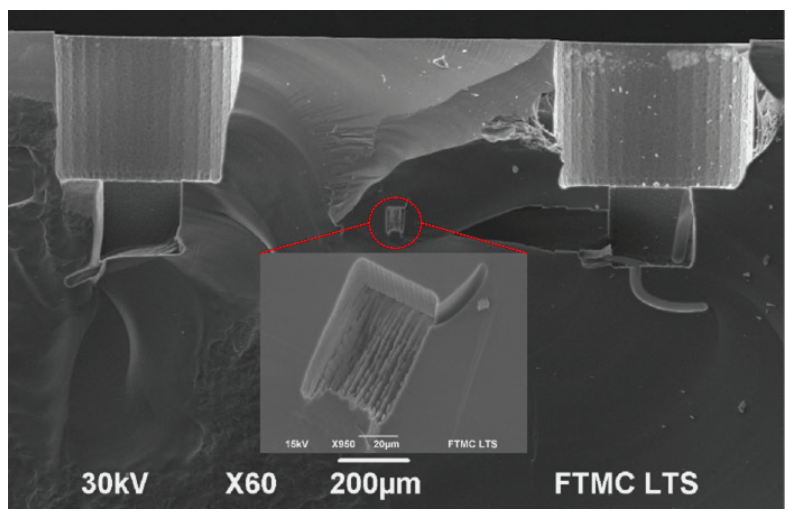

Fig. 7 SEM micrograph of the cross-section of the cleaved in yz plane micropump structure. The cleaved sample was coated of 5 $\mathrm{nm}$ gold layer for SEM analysis. 
Finally, the complex structure with the embedded rotator that rotates on its axis was fabricated. The device was designed in such a way as to compensate the taper angle of the micro channels emergent due to arising from the etching selectivity limitations. The etched structure was filled with acetone, and the optical microscope pictures were snapped at different time moments during acetone evaporation and show rotation of the microrotator due to capillary forces (figure 8). In the upper part of the microrotator the terminals that should be used as interconnection between micro to macro fluidic are formed.

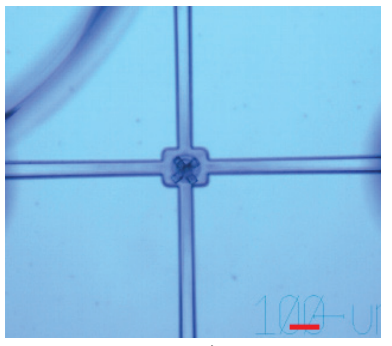

a)

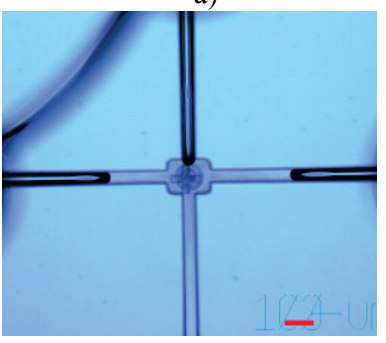

c)

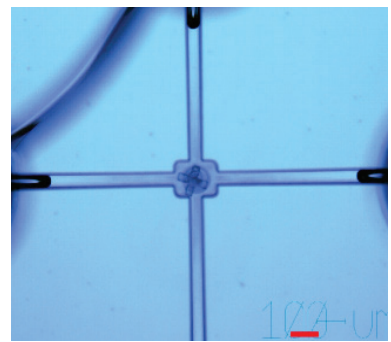

b)

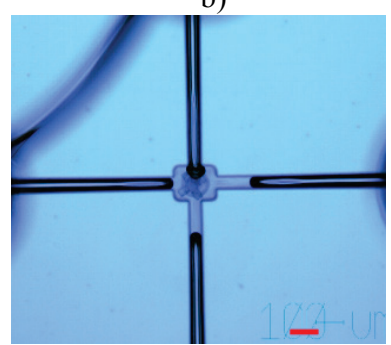

d)
Fig. 8 The microrotator embedded inside a fused silica and filled with acetone. a),b),c), d) - Acetone evaporation at different time moments. Dark lines show an empty channel. Red bar indicates $100 \mu \mathrm{m}$.

The needle with appropriate dimension was selected as an interconnector and it was sliced to the required length. The radius of the needle $(\sim 0.25 \mathrm{~mm})$ matched very well with the radius of the terminal and this facilitated the process of assembling. Epoxy glue was used for needle fixation. Fig. 9 shows the prepared embedded microrotator that was tested by interconnecting the microdevice terminal with the silicon hose and a syringe.

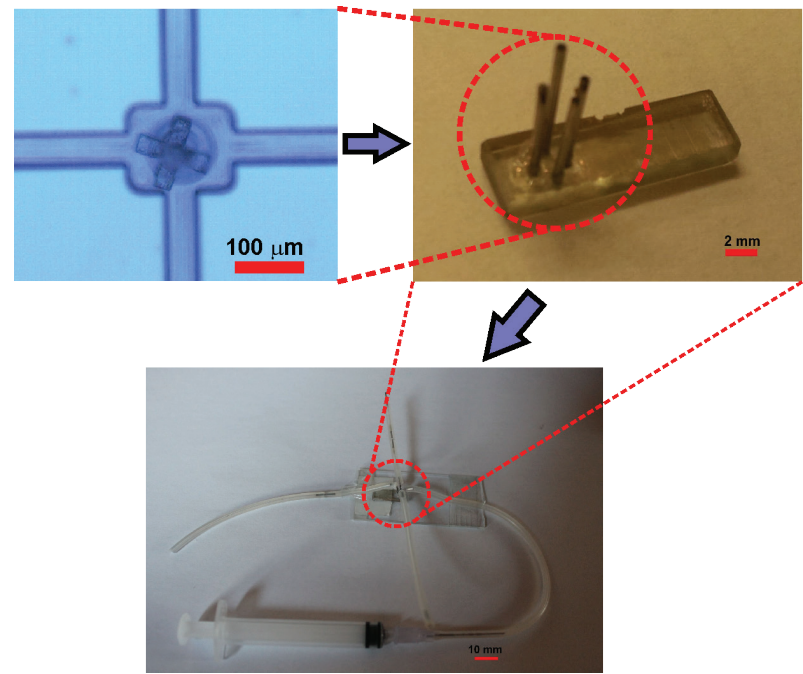

Fig. 9 Microrotator with needle-like interconnectors of $\sim 500 \mu \mathrm{m}$ diameter.
This microdevice has mechanical rotator from the same material (fused silica) embedded inside etched microchannels as opposed to the passive mixing devices [19].The further investigations to make proper interconnections are needed [20].

\section{Conclusions}

Two different scanning methods for the formation of three-dimensional microsystems were tested: the line scanning strategy and the contour scanning strategy. The etching selectivity with the proposed contour scanning shows promising results as enable easier acid penetration into modified region.

The formation of structures with different laser pulse energies, the pulse overlap, polarization states and distances between scanning lines was performed. The maximum etched volume speed for linear polarization was achieved with the $200 \mathrm{~nJ}$ pulse energy and for the circular polarization - with the $400 \mathrm{~nJ}$ pulse energy.

The volume etching rate with the linear polarization was larger when the pulse energy was decreased to $200 \mathrm{~nJ}$ that may be concerned with nano-gratings singularity dependence on the laser pulse energy. In the circular polarization case, the etching speed does not depend on the scanning direction. It has been shown that the circular polarization in combination with the contour scanning strategy is preferable when the complex 3D structures are formed.

The grain size of the etched structure can be minimized when the lower pulse energy for the formation of structures is used. In this case the surface roughness is minimized.

The micropump and microrotator devices were prepared and the fluid transportation possibility was tested. Further investigations of interconnections between micro and macro regions are required.

\section{References}

[1] Y. Bellouard, A. A. Said, M. Dugan and P. Bado, International Symposium on Optomechatronic Technologies (ISOT 2009), 21-23 September 2009, Istambul, (pp. 445450)

[2] Y. Bellouard, A. A. Said, P. Bado, Opt.Express13(2005) 6635.

[3] P. Yager, T. Edwards, E. Fu, K. Helton, K. Nelson, M.R. Tam, B.H. Weigl, Nature 442(2006) 412.

[4] P.S. Dittrich, K. Tachikawa, A. Manz, Anal. Chem.78(2006) 3887.

[5]Y. Cheng, K. sugioka, K. Midorikawa, and Z. Xu, Opt. Lett. 29(2004)2007.

[6] Z. Wang, K. Sugioka and K. Midorikawa, Appl. Phys. A: Mater. Sci. Process., 93(2008) 225.

[7] K. Sugioka and Y. Cheng, Lab Chip, 12 (2012)3576.

[8] G. D. Valle, R. Osellame and P. Laporta, J. Opt. A: Pure Appl. Opt. 11 (2009)013001.

[9] K.K.B.Hon, L. Li, I.M. Hutchings, CIRP Annals Manufacturing Technology 57 (2008) 601.

[10] R. Osellame, G. Cerullo, R. Ramponi, Femtosecond laser micromachining Photonic and microfluidic devices in transparent materials (Springer, Milano, 2012).

[11] A. Marcinkevičius, S. Juodkazis, M. Watanabe, M. Miwa, S. Matsuo and H. Misawa, Opt. Lett. 26(2001) 277. 
[12] Y. Bellouard, A. Said, M. Dugan and P. Bado, Opt. Express,12 (2004) 2120.

[13] S. Kiyama, S. Matsuo, S. Hashimoto, and Y. Morihira, J. Phys. Chem. C 113 (2009) 11560.

[14] V.Stankevič, G. Račiukaitis, Lithuanian Journal of Physics, 54 (3), 136-141, (2014).

[15] R. Taylor, C. Hnatovsky, E. Simova, Laser \& Photonics Rev. 2 (2008) 26.

[16] R.S. Taylor, E. Simova and C. Hnatovsky, Opt. Lett. 33 (2008) 1312.
[17]X. Yu, Y. Liao, F. He, B. Zeng, Y. Cheng, Z. Xu, K. Sugioka and K. Midorikawa, J. appl. phys. 109, (2011)053114.

[18] F. He, J. Lin, Y. Cheng, Appl. Phys. B 105 (2011) 379.

[19] Y. Liao, J. Song, E. Li, Y. Luo, Y. Shen, D. Chen, Y. Cheng, Z. Xu, K. Sugioka and K. Midorikawa, Lab Chip, 12, (2012) 746.

[20] Jr-H. Tsai and L Lin, J. Micromech. Microeng. 11 (2001) 577.

(Received: July 07, 2014, Accepted: November 14, 2014) 